\title{
Dairy producers value DHIA milk testing, but some deterred by cost
}

\author{
Gerald E. Higginbotham $\square \quad$ Steven L. Berry \\ Kenneth E. Lanka 口 William R. VerBoort \\ Robin Seldin $\square \quad$ Cathy Dei
}

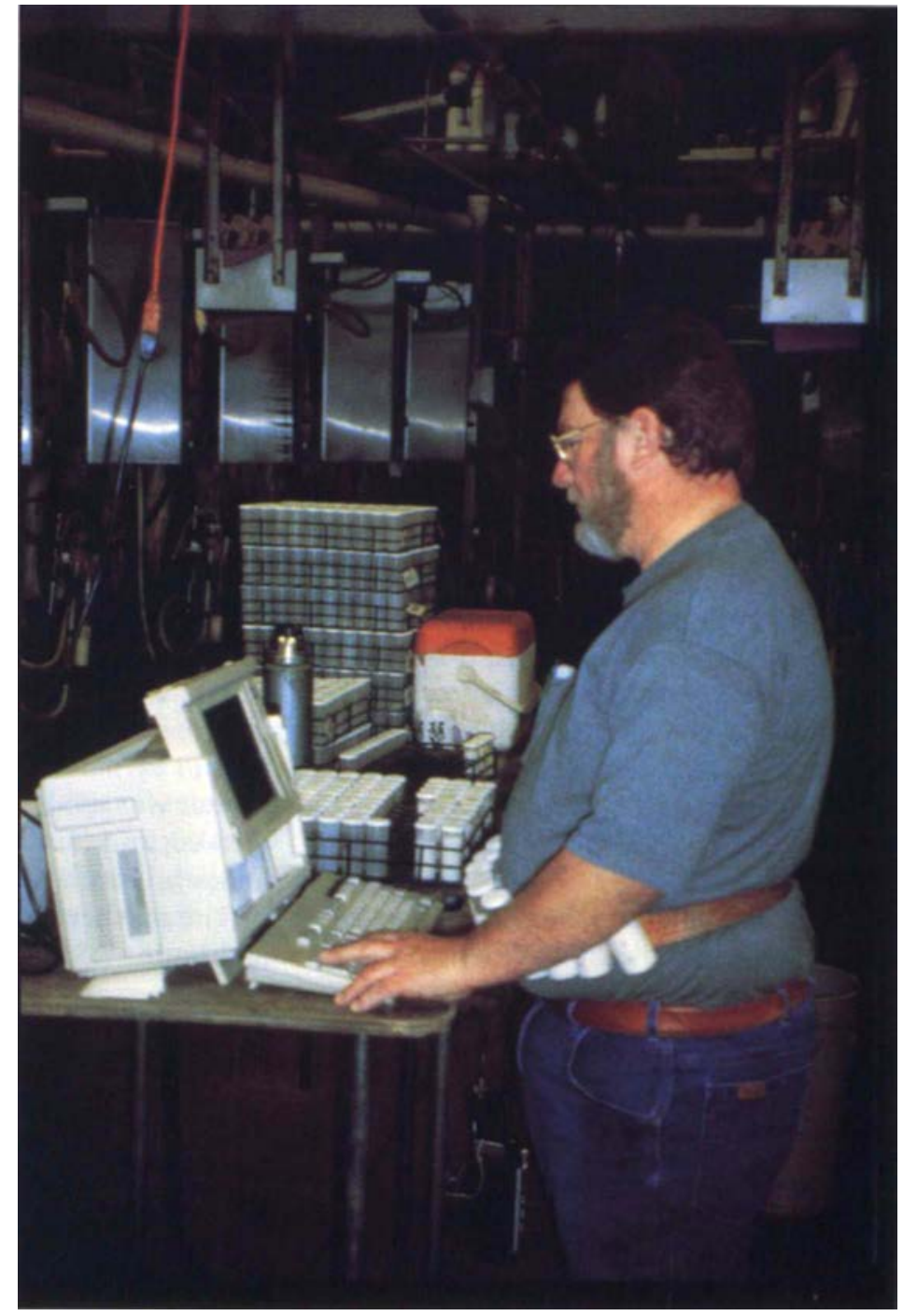

A survey was conducted to assess the attitudes of past and current members of the California Dairy Herd Improvement Association (CDHIA) toward the DHIA program. Members placed importance on service and production data. Northern and southern DHIA members rated their local DHIA supervisor high with regard to knowledge and service. All regions rated their local DHIA management high with regard to knowledge and service. Over $50 \%$ of the northern and southern DHIA members rated laboratory service high. All regions were satisfied with the service of the dairy records processing centers. Cost and the dairies' adoption of on-farm milkweight meters and/or computers were the most frequently cited reasons for discontinuing DHIA membership. Based on information collected in this survey, CDHIA members continue to look to DHIA records for production and management information.

\author{
Fresno DHIA su- \\ pervisor Terry Brown. \\ Service from local \\ DHIA supervisors is \\ an important consid- \\ eration for California \\ DHIA members.
}

duction of cows in untested herds.

The Dairy Herd Improvement Association (DHIA) program has been advocated as a management tool for efficient milk production since its inception in 1920. The nonprofit DHIA program provides dairy farmers with periodic (usually monthly) measurements of individual cow milk production, along with fat, protein or solidsnot-fat content. From the individual cow milk sample collected at the dairy, the DHIA lab also determines the somatic cell count (SCC) as well as fat and protein. The SCC is an indication of udder health. These measurements are valuable for making genetic and culling decisions. Several studies have compared the production of cows in DHIA-tested herds with pro-
Most recently, results from a National Animal Health Monitoring System survey showed herds to have higher rolling herd average milk production (yearly production per milking cow) when DHIA record-keeping systems are used.

In spite of the documented advantages of DHIA testing, some members become disenchanted with the program and leave. A survey of Wisconsin dairy

$\begin{aligned} & \text { TABLE 1. Mean herd size of dairies surveyed } \\
& \text { that were currently enrolled in DHIA programs }\end{aligned}$
\begin{tabular}{lrrrr}
\hline \hline & Northern & $\begin{array}{r}\text { Region } \\
\text { Central }\end{array}$ & Southern \\
\hline Herds, no. & 37 & 23 & 20 \\
Mean size & 215 & 700 & 1,182 \\
Range & $50-620$ & $130-2,375$ & $209-3,500$ \\
\hline
\end{tabular}


farmers revealed that a high proportion of respondents had participated in the DHIA program but had left it. Little information has been gathered on why farmers discontinue their membership. A survey of former members of the Missouri DHIA found that reasons for discontinuing membership included poor service, cost, dispersal sale, replacement with other records and no benefit received.

From 1990 to 1994, participation of California dairy farmers in the DHIA program increased from $63.2 \%$ to $66.7 \%$. In spite of this high level of DHIA membership in California, some dairy farmers have left the program. Information gathered from former members may help to redirect DHIA services to members and to prevent future losses of members. In addition, information from dairy farmers currently enrolled in DHIA testing programs may help to maintain or improve the quality of service provided. We surveyed past and present California DHIA (CDHIA) members to ascertain the strengths and weaknesses of the CDHIA.

The objectives of this study were (1) to provide information to local DHIAs

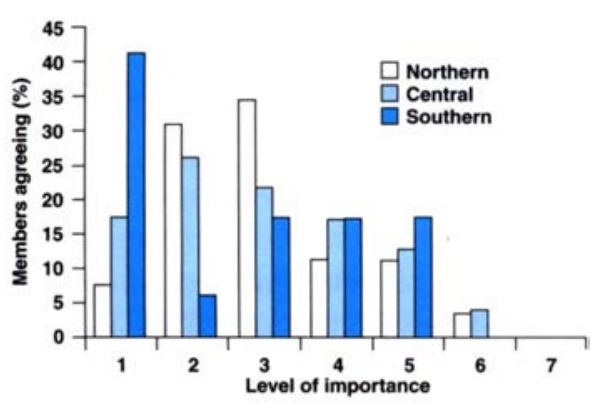

Fig. 1. Importance of service to CDHIA members on a 7-point scale $(1=$ most important, 7 = least important).

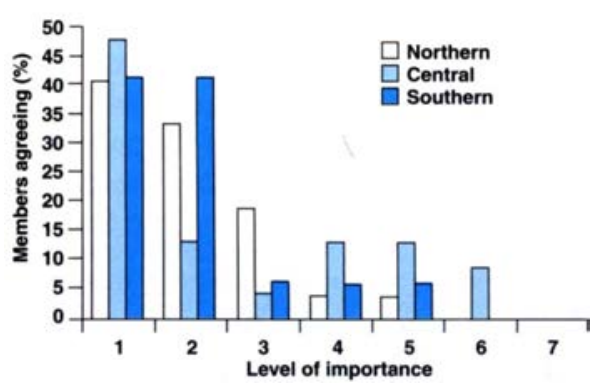

Fig. 2. Importance of production statistics to CDHIA members on a 7-point scale (1 = most important, 7 = least important). about reasons that dairy farmers are enrolled in the DHIA or have discontinued membership; and (2) to provide Cooperative Extension and DHIA personnel with directions for future educational and service activities.

\section{Survey dairies}

We randomly selected 60 California dairy farmers who were members of the DHIA as of August 1993 from each of three regions of California (northern, central and southern) Of the 180 selected dairy farmers, we actually surveyed $80-37$ in the northern region, 23 in the central region and 20 in the southern region. Reasons for not being able to contact all selected dairies were (1) inability to locate the manager or owner of dairy; (2) the dairy farmer was too busy to be interviewed; and (3) the dairy farm was no longer in business.

The northern and central regions had multiple local DHIAs, and the southern region had one local DHIA Mean herd size and range of dairies surveyed were typical for each geographical area (table 1).

An interviewer with a 27-question survey visited each dairy farmer. The questions were designed to elicit attitudes toward local DHIA supervisors and management, state DHIA management and dairy records processing centers. Other questions about dairy management were also presented.

In addition to current participants, we identified 30 dairies in each region that had discontinued DHIA services between 1990 and 1993, but were able to locate only 22 altogether. We interviewed these 22 dairy farmers with the same survey form but also asked about their reasons for leaving the DHIA and about the circumstances under which they might rejoin. Frequency distributions were generated for all qualitative answers.

\section{Dairies currently enrolled in DHIA}

The ages of dairy farmers who were interviewed ranged: $4.5 \%$ less than 25 years of age; $21.3 \%$ between 25 and 34 ; $34.8 \%$ between 35 and $44 ; 22.5 \%$ between 45 and 54 ; and $16.9 \%$ age 55 or older. Our results indicate that respon- dents in our study were younger than in the Ohio and Wisconsin studies. The researchers in Ohio and Wisconsin found that the DHIA program was being accepted more readily by younger dairy farmers than by older farmers. In the present study, $4.5 \%$ of the respondents had an eighth grade education, $46.6 \%$ had completed high school, $26.1 \%$ had some college education, and $22.7 \%$ had a college degree. The Ohio study had a lower percentage of high school graduates than our study.

\section{Participant responses to survey}

Participants were asked to rank the importance of DHIA functions on a seven-point scale ( $1=$ most important, $7=$ least important). Over $40 \%$ of DHIA members in the southern region ranked service as most important (fig. 1). The DHIA members in the northern and central regions also tended to place moderate to high importance on service from their local DHIA. Over $40 \%$ of DHIA members in all regions ranked production statistics from DHIA records as most important (fig. 2). Although many central region CDHIA members ranked production statistics as most important, some placed less importance on production statistics than members in the other two regions. When asked to rank the importance of timeliness of receiving DHIA information, all regions ranked it as medium in importance. Genetic information from DHIA was ranked as medium to low importance by all regions. Records of the DHIA for management use were fairly important for all regions, and all regions ranked awards as unimportant.

Respondents were asked to assess the knowledge and service of various aspects of the DHIA, ranking them on a five-point scale, from very low opinion to very high (table 2 ). To simplify discussion, we have combined the high and very high categories into a high category and the low and very low categories into a low category.

Southern DHIA members rated their DHIA supervisor highest with regard to knowledge and service. The DHIA members in the central and 
northern regions rated their supervisors lower. The DHIA members in all three regions generally ranked their local DHIA management high for knowledge and service. In the southern region, $95 \%$ of the members ranked their local DHIA management high with regard to knowledge and service. Over $60 \%$ of DHIA members in the northern and central regions rated the service of their local DHIA management as high.

In the southern region, $100 \%$ of the DHIA members rated the DHIA laboratory service as high. The DHIA members in the northern and central regions rated DHIA laboratory service somewhat lower, but less than $7 \%$ rated it as low. The central and southern regions rated their dairy records processing centers high for overall service; the northern region had a higher percentage of respondents who rated their dairy records processing center as medium.

From 55\% (southern) to $72 \%$ (northern) of the respondents said that they directed questions about DHIA to their local DHIA supervisor or management. Northern and central region members tended to use their DHIA supervisor or management more frequently than did southern region members.

We asked the dairy farmers who provided them with information and education on DHIA records. Approximately $75 \%$ of DHIA members in the northern region received information on DHIA records through their DHIA supervisor. Nearly $50 \%$ of members in the central region also reported that they receive their information about DHIA records through their DHIA supervisor. Southern DHIA members depend mostly on DHIA magazines and their local director for DHIA information. Because DHIA supervisors generally visit the same dairy each month, they have an opportunity to educate dairy farmers on the use and interpretation of records. Of the respondents, $18 \%$ to $28 \%$ said they received information concerning their DHIA records from their veterinarian; responses were similar for Cooperative Extension $(10 \%$ to $28 \%)$. Responses to this question reinforce the role of Cooperative Extension in some of the more traditional ways of extending knowledge, such as articles in newsletters, DHIA magazines and trade publications.

As a follow-up to the question about where members receive their DHIA information, dairy farmers were asked what kinds of DHIA educational programs are needed. Members in all regions indicated that newsletters are the most beneficial medium for disseminating DHIA records information. This preference was more apparent in the southern region, where more than $83 \%$ favored newsletters as a form of educational material. The DHIA members in the northern and central regions also desired newsletters and group meetings. In the northern region, $50 \%$ of the respondents felt that on-farm visits were an important source of education for them. Education about DHIA records is a high priority for local DHIA and Cooperative
Extension personnel. Respondents in Ohio research indicated that they thought DHIA and Cooperative Extension should conduct more educational meetings on the use and interpretation of DHIA records.

When asked about their reasons for joining DHIA, respondents indicated that management information was a major incentive for joining DHIA across all geographical regions (table 3). Milk components (fat, protein or solids-notfat content) and somatic cell counts were also important considerations. Use of DHIA records for genetic information was not given as a prime reason for joining the DHIA program, except in the northern region, where $36.1 \%$ of the respondents noted this as a reason for joining.

\section{Former DHIA members}

Because of difficulty in locating dairy farmers in each region who had discontinued the DHIA program, the

\begin{tabular}{|c|c|c|c|}
\hline \multirow[b]{3}{*}{ Item } & $\begin{array}{l}\text { n of the Dr } \\
\text { to respon }\end{array}$ & e and ser & \\
\hline & \multicolumn{3}{|c|}{ Responses } \\
\hline & $\begin{array}{c}\text { Northern } \\
\text { region }\end{array}$ & $\begin{array}{l}\text { Central } \\
\text { region }\end{array}$ & $\begin{array}{c}\text { Southern } \\
\text { region }\end{array}$ \\
\hline \multicolumn{4}{|c|}{ DHIA supervisor } \\
\hline High & 65.7 & 62.1 & 90.0 \\
\hline Medium & 27.7 & 27.6 & 10.0 \\
\hline Low & 5.6 & 10.3 & 0.0 \\
\hline \multicolumn{4}{|c|}{ Local DHIA management } \\
\hline High & 63.9 & 60.0 & 95.0 \\
\hline Medium & 36.1 & 23.3 & 5.0 \\
\hline Low & 0.0 & 16.7 & 0.0 \\
\hline \multicolumn{4}{|c|}{ California Dairy Herd Improvement Association } \\
\hline High & 70.6 & 46.7 & 89.4 \\
\hline Medium & 26.4 & 33.3 & 5.3 \\
\hline Low & 3.0 & 20.0 & 5.3 \\
\hline \multicolumn{4}{|c|}{ DHIA laboratory } \\
\hline High & 67.6 & 68.9 & 100.0 \\
\hline Medium & 29.7 & 24.2 & 0.0 \\
\hline Low & 2.7 & 6.9 & 0.0 \\
\hline \multicolumn{4}{|c|}{ Dairy records processing center } \\
\hline High & 72.2 & 90.0 & 94.7 \\
\hline Medium & 25.0 & 6.7 & 5.3 \\
\hline Low & 2.8 & 3.3 & 0.0 \\
\hline
\end{tabular}

\begin{tabular}{lccc} 
& \multicolumn{2}{c}{ TABLE 3. Reasons for joining DHIA according to survey responses } \\
\hline \hline \multirow{2}{*}{ Item } & \multicolumn{3}{c}{ Region } \\
\cline { 2 - 4 } & Northern & Central & Southern \\
\hline & 66.7 & 37.9 & 45.0 \\
Milk components & 58.3 & 34.5 & 35.0 \\
Somatic cell count & 69.4 & 75.9 & 70.0 \\
Management information & 36.1 & 24.1 & 15.0 \\
Genetic information & 13.9 & 20.7 & 30.0
\end{tabular}




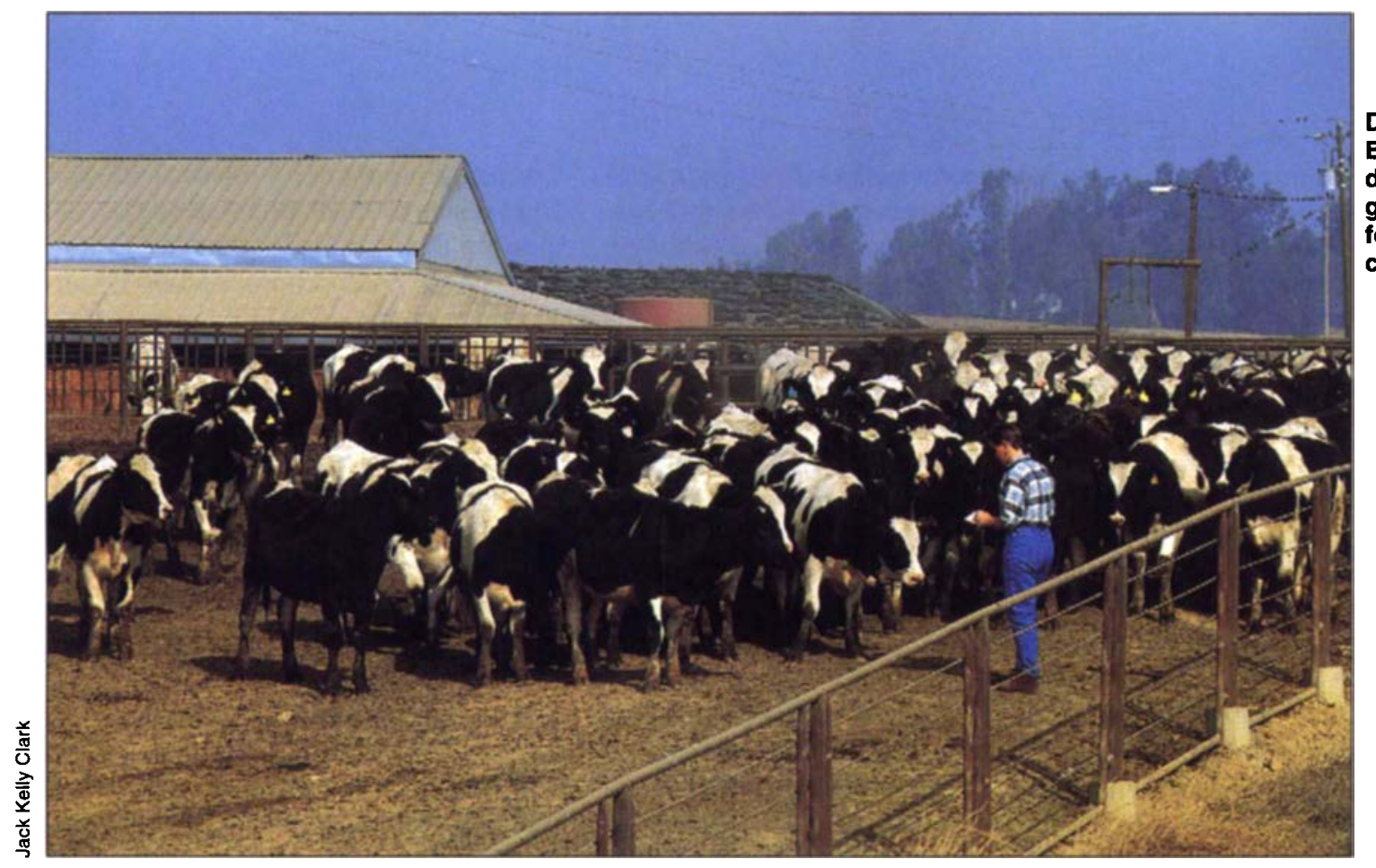

Dairy advisors like Barbara Reed use production information gathered by the DHIA for research and education.

22 former DHIA members from all regions were pooled. Reasons given for discontinuing the program were supervisor competence; cost; the use of on-farm milk-weight meters and/or computers; local DHIA management; did not use the records; and others. Over $55 \%$ of those surveyed cited cost as a major determinant for leaving the DiHIA program, and over $30 \%$ cited on-farm meters and/or computers as the reason.

A similar survey of DHIA members in Missouri showed that $25.4 \%$ of those who discontinued DHIA programming reported it to be too costly. Respondents to one Ohio survey felt that DHIA programs were providing more information than they could use and that the cost of the program should be reduced. In a subsequent questionnaire by the same researchers, respondents who strongly agreed that DHIA was cost effective had larger gains in production per cow $(1,795$ pounds) during a 7-year period than those respondents who were neutral (559 pounds) or who strongly disagreed (139 pounds).

Milk production figures for herds participating in our survey were not recorded.

\section{Conclusions}

Based on the information collected in this survey, we concluded that
CDHIA members continue to look to DHIA records for production and management information. Particularly among CDHIA members in the southern region, service from the DHIA is important. Most local DHIAs recognize outstanding herds through an awards process; however, our survey indicated that most dairy farmers ranked awards as a low priority.

Dairy farmers from all regions rated their local DHIA management and DHIA supervisor high with regard to knowledge and service. The DHIA supervisors are also regarded as a major source of DHIA information. Dairy farmers enrolled in DHIA in all regions felt that newsletters from their local DHIA would be beneficial.

Those dairy producers who left the DHIA cited cost as a major factor in their decision to leave. When asked what would influence them to rejoin DHIA, a reduction in cost was mentioned most often. The second most common reason for discontinuing DHIA testing was the use of on-farm milk meters and/or computers, which would indicate that dairy farmers are either not perceiving the added value of milk component and somatic cell count information or are getting the information in some other, more economical way.

To retain DHIA members, local associations and Cooperative Extension personnel should collaborate to educate DHIA members on the economic benefits of being in the DHIA program. Cooperative Extension encourages DHIA membership because it uses production information gathered by the program for education, research and genetic evaluation to help dairies manage their operations more efficiently. Efforts should also be made to ascertain what other services dairy farmers need that could be provided by DHIA. The DHIA is undergoing rapid evolution and must either adapt or members will find a more cost-effective means of getting the information and services they need.

G.E. Higginbotham is Dairy Advisor, UC Cooperative Extension, Fresnol Madera counties; S.L. Berry is Extension Dairy Management and Health Specialist, Department of Animal Science, UC Davis; K.E. Lanka is former Dairy Advisor, UC Cooperative Extension, Stanislaus/Merced counties; W.R. VerBoort is General Manager, California Dairy Herd Improvement Association, Fresno; R. Seldin is Executive Vice President, AgriTech Analytics, Tulare; and C. Dei is Field Representative, DHIProvo, Provo, Utah.

The authors wish to acknowledge financial support from the California Dairy Herd Improvement Association. 